\title{
Kernel Principal Component Analysis for UWB-Based Ranging
}

\author{
Vladimir Savic, Erik G. Larsson, Javier Ferrer-Coll and Peter Stenumgaard
}

\section{Linköping University Post Print}

\section{Tweet}

N.B.: When citing this work, cite the original article.

Original Publication:

Vladimir Savic, Erik G. Larsson, Javier Ferrer-Coll and Peter Stenumgaard, Kernel Principal Component Analysis for UWB-Based Ranging, 2014, IEEE International Workshop on Signal Processing Advances in Wireless Communications (SPAWC).

C2014 IEEE. Personal use of this material is permitted. However, permission to reprint/republish this material for advertising or promotional purposes or for creating new collective works for resale or redistribution to servers or lists, or to reuse any copyrighted component of this work in other works must be obtained from the IEEE.

http://www.spawc2014.org/

Postprint available at: Linköping University Electronic Press http://urn.kb.se/resolve?urn=urn:nbn:se:liu:diva-106648 


\title{
Kernel Principal Component Analysis for UWB-Based Ranging
}

\author{
Vladimir Savic ${ }^{1}$, Erik G. Larsson ${ }^{1}$, Javier Ferrer-Coll ${ }^{2}$, and and Peter Stenumgaard ${ }^{3}$ \\ ${ }^{1}$ Dept. of Electrical Engineering (ISY), Linköping University, Sweden \\ ${ }^{2}$ Dept. of Electronics, Mathematics and Natural Sciences, University of Gävle, Sweden \\ ${ }^{3}$ Swedish Defense Research Agency (FOI), Sweden \\ Emails: vladimir.savic@liu.se, erik.larsson@isy.liu.se, javier.ferrercoll@hig.se, peter.stenumgaard@foi.se
}

\begin{abstract}
Accurate positioning in harsh environments can enable many application, such as search-and-rescue in emergency situations. For this problem, ultra-wideband (UWB) technology can provide the most accurate range estimates, which are required for range-based positioning. However, it still faces a problem in non-line-of-sight (NLOS) environments, in which range estimates based on time-of-arrival (TOA) are positively biased. There are many techniques that try to address this problem, mainly based on NLOS identification and NLOS error mitigation. However, these techniques do not exploit all available information from the UWB channel impulse response. In this paper, we propose a novel ranging technique based on kernel principal component analysis (KPCA), in which the selected channel parameters are projected onto nonlinear orthogonal highdimensional space, and a subset of these projections is then used for ranging. We tested this technique using UWB measurements obtained in a basement tunnel of Linköping university, and found that it provides much better ranging performance comparing with standard techniques based on PCA and TOA.

Index Terms - ranging, ultra-wideband, time-of-arrival, kernel principal component analysis, machine learning.
\end{abstract}

\section{INTRODUCTION}

UWB technology [1] represents a promising technology for many applications including range-based positioning in harsh environments. A large bandwidth of the UWB signal provides a high resolution for TOA-based ranging, and allows propagation through the thin obstacles. However, it still faces a problem in NLOS environments caused by thick obstacles, in which TOA-based range estimates are positively biased. This is especially the problem in environments such as tunnels, factories and urban canyons. Consequently, a positioning system based on these range estimates would provide very poor performance.

There are many techniques [2] that try to address this problem, and they are mainly based on NLOS identification and NLOS error mitigation. However, these techniques do not exploit all available information from the UWB channel impulse response. In this paper, we propose a novel technique that uses all available channel parameters and does not require NLOS identification and NLOS error mitigation. We adapt a nonlinear version of principal component analysis (PCA) (known as kernel PCA (kPCA)) [3], in which the selected channel parameters are projected onto nonlinear orthogonal high-dimensional space, and a subset of these projections is then used for ranging. Although popular for computer vision problems, to the best of our knowledge, this technique is not so far used for UWB-based ranging. We tested this technique using UWB measurements obtained in a basement tunnel of Linköping university [4], and found that it provides much better ranging performance comparing with standard techniques based on PCA and TOA. The unique drawback of this approach is high computational complexity.

The remainder of this paper is organized as follows. In Section II, we present an overview of other UWB-based ranging techniques. In Section III, we review the generic approach for TOA-based ranging with NLOS identification and NLOS error mitigation. Then, in Section IV, we propose a novel ranging technique based on kPCA. Experimental results, using UWB measurements obtained in tunnel environment, are provided in Section V. Finally, Section VI summarizes our conclusions and provides proposals for future work.

\section{RELATED WORK}

UWB-based ranging is typically performed using TOA estimates since it allows very fine resolution. However, with NLOS measurements, this approach leads to positively biased estimates. There are many proposals in literature for how to deal with this problem [2], which are mainly based on NLOS identification and error mitigation. NLOS identification attempts to distinguish between LOS and NLOS conditions, while NLOS error mitigation attempts to reduce the error caused by an NLOS condition (assuming that this NLOS condition is identified).

NLOS identification could be carried out by analyzing the variance of the time-series of the range estimates [5]. Since the NLOS measurements usually have much larger variance, the hypothesis testing can be easily performed. However, this approach would lead to high latency since it requires a large number of measurements. An alternative approach is to use channel parameters from the power delay profile (PDP), which is obtained from the channel impulse response. For instance, in [6] three parameters are jointly used (RMS delay-spread, TOA and received signal stregth (RSS)) to distinguish between LOS and NLOS scenarios. They found that RMS delay spread is the most useful for this problem, but the combination of these three parameters can improve the probability of correct 
identification. In [7], the authors found that the kurtosis provides consistent information about NLOS condition, and that using multiple antennas can improve this information. In [8], multiple parameters are considered by using a nonparametric least-square support-vector-machine (LS-SVM) classifier. This approach does not require statistical models, since it directly works with training samples. A nonparametric approach is also used in [9], where the authors use the training samples to construct the kernel of the LOS and NLOS error probability density functions (PDFs). Then, they use Kullback-Leibler (KL) divergence to measure the distance between these PDFs, and set the decision threshold.

Once NLOS identification is performed, the measurement can be discarded but it would lead to unnecessary loss of useful information (especially, if there are no sufficient LOS links). Therefore, NLOS error mitigation is required to make NLOS measurements useful for ranging. Since the distribution of the NLOS error depends on the spatial distribution of the scatterers, the mitigation could be performed by modeling these scatterers [10]. However, this approach is typically not feasible due to the complex shape of the environment, and possible dynamic obstacles. Another way is to model the NLOS error as a function of some channel parameter. For example, in [11], the authors found that the NLOS error is increasing with the mean excess delay and the RMS delay spread. Therefore, a simple polynomial model can be used to significantly reduce this error. In many cases, it may not be possible to detect an NLOS condition, but only its probability. In that case, a soft-decision approach is required, in which NLOS identification and error mitigation are combined into one single step. This approach is proposed in [12], in which the ranging likelihood function is a mixture of LOS and NLOS models. Finally, nonparametric (kernel-based) regression can be also used to compute the NLOS error as a function of multiple channel propagation parameters. For this purpose, LSSVM regression has been used in [8], and Gaussian process regression in [13]. They are capable to provide NLOS ranging error without explicit NLOS identification.

\section{TOA-BASED RANGING AND ERROR MITIGATION}

Given complex impulse responses of the channel, $h(t)=$ $\sum_{k} a_{k} \delta\left(t-\tau_{k}\right)\left(a_{k} \in \mathbb{C}\right)$, we can obtain the power delay profile (PDP) as $|h(t)|^{2}$. However, since most of the components of the PDP are typically caused by thermal noise, we consider only components above a certain threshold $P_{T H}[\mathrm{dBm}]$, i.e.,

$$
p_{h}(t)= \begin{cases}|h(t)|^{2}, & \text { if } 10 \log _{10}\left(|h(t)|^{2} / P_{0}\right)>P_{T H} \\ 0, & \text { otherwise }\end{cases}
$$

where $P_{0}=1 \mathrm{~mW}$. There are many ways to choose the threshold [14], but we choose the value which provides the best trade-off between false-alarm and missed-detection rates. Then, we can extract a number of channel parameters from PDP, such as TOA, RSS, RMS delay spread, etc. See [4, Section IV] for more details.

The range estimate can be then easily determined as $\hat{d}_{T O A}=c \tau_{1}$ where $\tau_{1}$ is estimated TOA, and $c=3 \cdot 10^{8} \mathrm{~m} / \mathrm{s}$ is the speed of light. However, this would lead to a large positive bias in the NLOS scenario. Therefore, NLOS identification and error mitigation are required to reduce this bias.

Here we describe the generic approach, based on selected channel parameters from the PDP. We define a binary variable $H \in\{\mathrm{LOS}, \mathrm{NLOS}\}$, and consider the following model:

$$
c \tau_{1}= \begin{cases}d+\mu_{L}+\nu_{L}, & \text { if } H=\operatorname{LOS} \\ d+g\left(\alpha_{E}\right)+\nu_{N}, & \text { if } H=\operatorname{NLOS}\end{cases}
$$

where $d$ is the true distance between transmitter and receiver, $\nu_{L}$ and $\nu_{N}$ are LOS and NLOS noise components $\left(\nu_{L} \sim p_{L}(\cdot)\right.$, $\left.\nu_{N} \sim p_{N}(\cdot)\right)$, respectively, and $\mu_{L}$ is a known LOS bias (caused by finite bandwidth, false alarms, etc.) $g\left(\alpha_{e}\right)$ is NLOS error modeled as (potentially, nonlinear) function of the selected channel parameter $\alpha_{E}$ from PDP $\left(\alpha_{E}\right.$ should be chosen empirically so that it has strong correlation with NLOS error). In order to estimate $H$, we use Bayes' rule:

$$
p\left(H \mid \alpha_{I}\right)=\frac{p\left(\alpha_{I} \mid H\right) p(H)}{\sum_{H^{\prime} \in\{\mathrm{LOS}, \mathrm{NLOS}\}} p\left(\alpha_{I} \mid H^{\prime}\right) p\left(H^{\prime}\right)}
$$

where $p(H)$ is the prior, and $p\left(\alpha_{I} \mid H\right)$ is the likelihood function. The prior may be chosen based on the geometry of the area, or set to non-informative state. The model for likelihood depends on the chosen channel parameter $\alpha_{I}$ and can be obtained using training data $\left(\alpha_{I}\right.$ should be chosen empirically so that it can well distinguish the LOS and NLOS states).

The likelihood function for range estimation is given in the mixture form:

$$
\begin{array}{r}
p\left(\tau_{1}, \alpha_{I}, \alpha_{E} \mid d\right)=p\left(H=\operatorname{LOS} \mid \alpha_{I}\right) p_{L}\left(c \tau_{1}-\mu_{L}-d\right)+ \\
p\left(H=\mathrm{NLOS} \mid \alpha_{I}\right) p_{N}\left(c \tau_{1}-g\left(\alpha_{E}\right)-d\right)(4)
\end{array}
$$

This (non-Gaussian) likelihood represents full statistical information about unknown distance, and can be used for probabilistic (typically, Bayesian) positioning algorithms. Assuming that $p_{L}(\cdot)$ and $p_{N}(\cdot)$ are Gaussian, a minimum mean-squareerror (MMSE) estimate of the distance is given by:

$$
\begin{aligned}
\hat{d}_{T O A, M}= & p\left(H=\operatorname{LOS} \mid \alpha_{I}\right)\left(c \tau_{1}-\mu_{L}\right)+ \\
& p\left(H=\operatorname{NLOS} \mid \alpha_{I}\right)\left(c \tau_{1}-g\left(\alpha_{E}\right)\right)
\end{aligned}
$$

There are many variations of this approach that will not be described here. For instance, it is possible to use multiple channel parameters for NLOS identification and error mitigation, but this would likely lead to over-counting of the same information since the parameters are typically correlated [4, Table III]. We are going to address this problem in the following section.

\section{KPCA-BASED RANGING}

Our goal is to perform ranging using all available channel parameters from PDP, and without NLOS identification and error mitigation. Given a channel parameter $\alpha_{k}(k=1, \ldots, K)$, we first transform it to centered and dimension-less data:

$$
a_{k}=\frac{\alpha_{k}-\mu_{\alpha_{k}}}{\sigma_{\alpha_{k}}}
$$



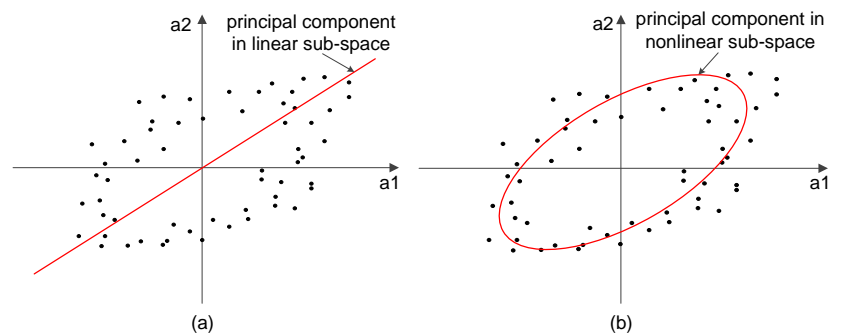

Fig. 1: Principal components of 2D data in (a) linear and, (b) nonlinear subspace. The latter one is much more accurate projection.

where $\mu_{\alpha_{k}}$ and $\sigma_{\alpha_{k}}$ are the mean and the standard deviation of $\alpha_{k}$, respectively. Then, we gather all transformed channel parameters into one vector $\mathbf{a}=\left(a_{1}, \ldots, a_{K}\right)^{T}$. Since elements of $\mathbf{a}$ are correlated ${ }^{1}$ with each other, we could apply eigenvalue decomposition, and then retain $M$ largest eigenvalues and corresponding eigenvectors, that can be used for ranging. This procedure is well-known as principal component analysis (PCA). However, PCA projects the data in a linear sub-space, so it becomes invalid if the data lies on nonlinear manifold. An example for 2D data is shown in Fig. 1. Therefore, we apply kernel PCA (kPCA) initially proposed in [3], which is capable to project the data to an arbitrary nonlinear manifold.

We first define nonlinear transformation $\phi(\mathbf{a})$, which transforms $K$-dimensional vector a to $N$-dimensional vector in a feature space. The feature space has large (possibly, infinite) dimensionality $(N>>K)$. For now, we also assume that $\phi(\mathbf{a})$ is zero-mean, so that $N \times N$ covariance matrix is given by $\mathbf{C}=E\left(\phi(\mathbf{a}) \phi(\mathbf{a})^{T}\right)$. Then, we could find the principal components using eigenvalue expansion of $\mathbf{C}$, but this is not possible to achieve explicitly since the feature space has high dimensionality. However, it can be shown [15, Chapter 12] that eigenvalue decomposition depends on $\phi(\mathbf{a})$ only via inner product $k\left(\mathbf{a}, \mathbf{a}_{\mathbf{n}}\right)=\phi(\mathbf{a})^{\mathbf{T}} \phi\left(\mathbf{a}_{\mathbf{n}}\right)$ (where $\mathbf{a}_{n}$ is a training sample), known as a kernel function. Thus, we find principal components using the following eigenvalue expansion:

$$
\mathbf{K} \mathbf{v}_{n}=\lambda_{n} N \mathbf{v}_{n}
$$

where $\mathbf{K}$ is an $N \times N$ kernel matrix with $i$-th row and $n$ th column given by $k\left(\mathbf{a}_{i}, \mathbf{a}_{n}\right)$ (i.e., kernel function computed for all possible pairs of the training samples). The eigenvalues and eigenvectors of $\mathbf{K}$ are given by $\lambda_{n} N$ and $\mathbf{v}_{n}$, respectively. Then, the projection of point a onto eigenvector $i$ is given by

$$
y_{i}(\mathbf{a})=\sum_{n=1}^{N} v_{i n} k\left(\mathbf{a}, \mathbf{a}_{n}\right)
$$

Therefore, we can obtain all projections using kernel functions, without explicit work in feature space. In practice, the feature vector is not zero-mean, but it only means that we need to

\footnotetext{
${ }^{1}$ Note that the point estimate of the distance can be directly found from correlated data (e.g., using LS-SVM). However, our main goal is to provide full statistical information about unknown distance.
}

replace $\mathbf{K}$ with a Gram matrix $\tilde{\mathbf{K}}$ :

$$
\tilde{\mathbf{K}}=\mathbf{K}-\mathbf{1}_{N} \mathbf{K}-\mathbf{K} \mathbf{1}_{N}+\mathbf{1}_{N} \mathbf{K} \mathbf{1}_{N}
$$

where $\mathbf{1}_{N}$ is the $N \times N$ matrix with all values equal to $1 / N$. We see that this matrix can be also computed without the feature vectors. Therefore, for kPCA, we only need to define a kernel function. A popular choice for kernel $^{2}$ is the polynomial kernel in the form:

$$
k\left(\mathbf{a}, \mathbf{a}_{n}\right)=\left(\mathbf{a}^{T} \mathbf{a}_{n}+1\right)^{c}
$$

where $c \in \mathbb{N}$ is the degree of polynomial that can be found empirically. Note that the standard PCA is a special case if we use a linear kernel $k\left(\mathbf{a}, \mathbf{a}_{n}\right)=\mathbf{a}^{T} \mathbf{a}_{n}$.

Then, given $M$ principal components $y_{i}(\mathbf{a})$ corresponding to $M$ largest eigenvalues, we need to provide a model that will relate them with the unknown range. In general case, this relationship is unknown, but we assume that appropriate kernel will make this relationship approximately linear ${ }^{3}$. Therefore, we assume that projected features $y_{i}=y_{i}(\mathbf{a})$ are linear functions of the true distance:

$$
y_{i}=b_{1, i} d+b_{0, i}+\nu_{y, i}
$$

where $b_{1, i}$ and $b_{0, i}$ are parameters that can be found using least-square curve fitting (using the same training samples used for kPCA), and $\nu_{y, i}$ is a noise component. Since the distribution of the noise is unknown, we assume that it is zero-mean Gaussian as the distribution that maximizes the entropy (given variance $\sigma_{y, i}^{2}$, found from the training samples). Assuming that we retained $M$ principal components, the likelihood function is given by:

$$
\begin{gathered}
p\left(y_{1}, \ldots, y_{M} \mid d\right)=\prod_{i=1, \ldots, M} p\left(y_{i} \mid d\right)= \\
\prod_{i=1, \ldots, M} \mathcal{N}\left(y_{i}-b_{1, i} d-b_{0, i} ; 0, \sigma_{y, i}^{2}\right)= \\
\prod_{i=1, \ldots, M} \mathcal{N}\left(d ; \frac{y_{i}-b_{0, i}}{b_{1, i}}, \frac{\sigma_{y, i}^{2}}{b_{1, i}^{2}}\right)
\end{gathered}
$$

Maximum number of principal components is equal to total number of the training samples $(N)$, which is much higher than dimensionality of the original data $(K)$. Since $M$ is expected to be larger than $K$, kPCA is typically used for nonlinear feature extraction, in contrast to PCA, which is typically used for dimensionality reduction. However, an efficient kernel would allow to use only very few components.

From (12), we can find the MMSE estimate of the distance:

$$
\hat{d}_{k P C A}=\left(\sum_{i=1}^{M} \frac{b_{1, i}^{2}}{\sigma_{y, i}^{2}}\right)^{-1} \sum_{i=1}^{M}\left(b_{1, i} \frac{y_{i}-b_{0, i}}{\sigma_{y, i}^{2}}\right)
$$

Recall that this estimate is valid for both LOS and NLOS scenarios, and is expected to provide much more accurate estimates. On the other hand, the complexity is much higher since we have to store $O\left(N^{2}\right)$ samples and to perform $O\left(M N^{2}\right)$

\footnotetext{
${ }^{2}$ Other widely used kernel (Gaussian) would lead to worse performance.

${ }^{3}$ Nonlinear model would be redundant since we already had opportunity to perform arbitrary nonlinear transformation with kPCA.
} 
TABLE I: Main measurement parameters

\begin{tabular}{ll}
\hline \hline Signal power & $12 \mathrm{dBm}$ \\
Waveform & sinusoidal \\
Center frequency & $3.5 \mathrm{GHz}$ \\
Bandwidth & $2 \mathrm{GHz}$ \\
Number of points & 3001 \\
Sweep time & $263 \mathrm{~ms}$ \\
Time resolution & $0.5 \mathrm{~ns}$ \\
Bandpass filter & Hann window \\
\hline \hline
\end{tabular}

operations for kPCA. If the number of samples $N$ is on the order of thousands, this should not be a problem with nowadays computational resources. Regarding the communication cost, kPCA requires transmission of the same data ( $K$ channel parameters) as PCA.

\section{EXPERIMENTAL RESULTS}

In this section, we analyze the proposed algorithm using UWB measurements obtained in a basement tunnel of Linköping university in Sweden.

\section{A. Measurement setup}

We used the measurement setup that consists of a vector network analyzer (VNA), two UWB omni-directional antennas and coaxial cables to connect the antennas with the VNA. A PC is used to set the VNA parameters and extract the multiple frequency responses from the instrument. In our case, we use a swept-frequency sinusoidal signal (with 3001 points) to characterize the channel between 2.5 and $4.5 \mathrm{GHz}$. The power level was set to $12 \mathrm{dBm}$, and a calibration of the system is performed to compensate for the effect of VNA, cables and antennas. Then, the frequency responses are transferred to the PC where a Hann window is used to reduce the out-of-band noise. Finally, by applying the Inverse Fast Fourier Transform (IFFT), the complex impulse responses are estimated, and subsequently, PDPs are calculated. We summarize the main parameters in Table I.

We decided to consider four different scenarios: LOS, and NLOS caused by three different obstacles: a metal sheet, a person, and a tunnel wall. For each of these scenarios, we placed the transmitter (Tx) in 3 positions and receiver $(\mathrm{Rx})$ in 30 locations forming the route through the tunnel. For each TxRx pair, we obtained 10 PDPs, so we obtained 3600 PDPs in total (900 per scenario). Since our initial analysis showed that thin obstacles (metal sheet and person) provide results similar as LOS, we will consider them as LOS. Therefore, we have available 2700 LOS samples and 900 NLOS samples. Half of these samples (1350 LOS samples and 450 NLOS samples) will be used as training data, and the rest will be used to test ranging performance.

The illustration of LOS experiments is shown Fig. 2.

\section{B. Performance analysis}

Our goal is to analyze the ranging error of the proposed approach and compare it with TOA-based techniques. For

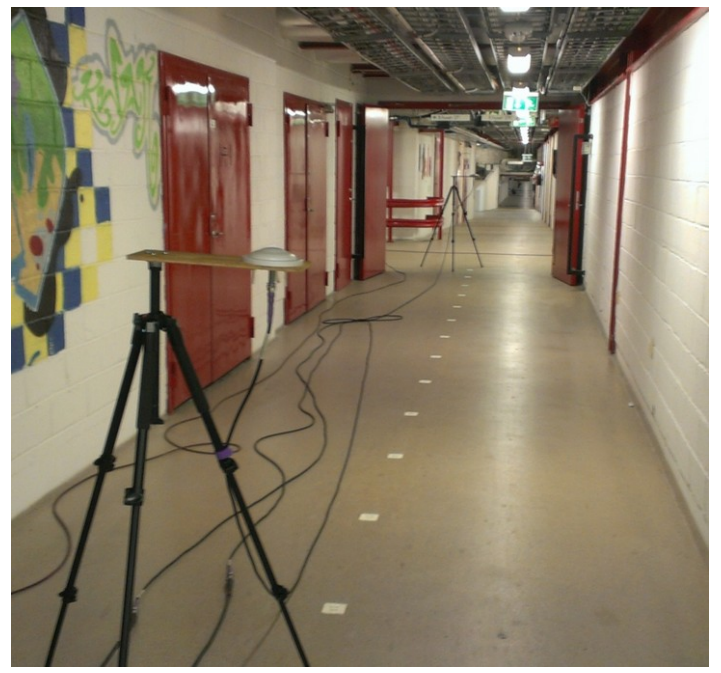

Fig. 2: Illustration of LOS experiments in LiU tunnel.

PCA/kPCA, we extract $K=8$ relevant channel parameters from PDP (given by [4, eqs. (2)-(9)]): TOA, RSS, maximum received power, mean excess delay, maximum excess delay, RMS delay spread, rise time and kurtosis. For TOA with NLOS identification and error mitigation, we found that rise time is the best channel parameter $\left(\alpha_{I}\right)$ for NLOS identification, and maximum excess delay is the best channel parameter $\left(\alpha_{E}\right)$ for NLOS error mitigation. Regarding the model in (2), we found that $\nu_{L}$ and $\nu_{N}$ approximately follow a zeromean Gaussian distribution, while $g\left(\alpha_{E}\right)$ can be modeled with second-order polynomial function. Likelihood function $p\left(\alpha_{I} \mid H\right)$ is assumed to follow exponential distribution with different parameter for LOS and NLOS scenario, and the prior $p(H)$ is non-informative. Numerical values of all parameters are available in [4, Table IV].

In order to empirically determine the optimal degree of polynomial kernel, we analyze root-mean-square error (RMSE) of the range estimates as a function of degree $c$, for different number of retained principal components $M$. We do not consider cases with $M>100$ since there are no more useful principal components (i.e., with non-negligible eigenvalues). According to Fig. 3, we see that the best performance is achieved for $c=3$ and $M \geq 40$. We also note that wrong value of the polynomial degree (e.g., $c=2$ ) could lead to significant loss in performance. Therefore, we choose thirddegree polynomial for further analysis.

Given optimal degree, we compare kPCA (for three representative values of $M$ ) with linear PCA, and two TOA-based techniques described in Section III. Cumulative distribution function $(\mathrm{CDF})$ of the ranging error is shown in Fig. 4. We can conclude the following:

- TOA-only techniques provides very accurate results for about $75 \%$ of the samples (i.e., LOS samples), when the performance is better than kPCA. However, NLOS samples can cause huge (up to $10 \mathrm{~m}$ ) error.

- TOA with mitigation can reduce the error caused by NLOS samples for about $1 \mathrm{~m}$, but it performs slightly 


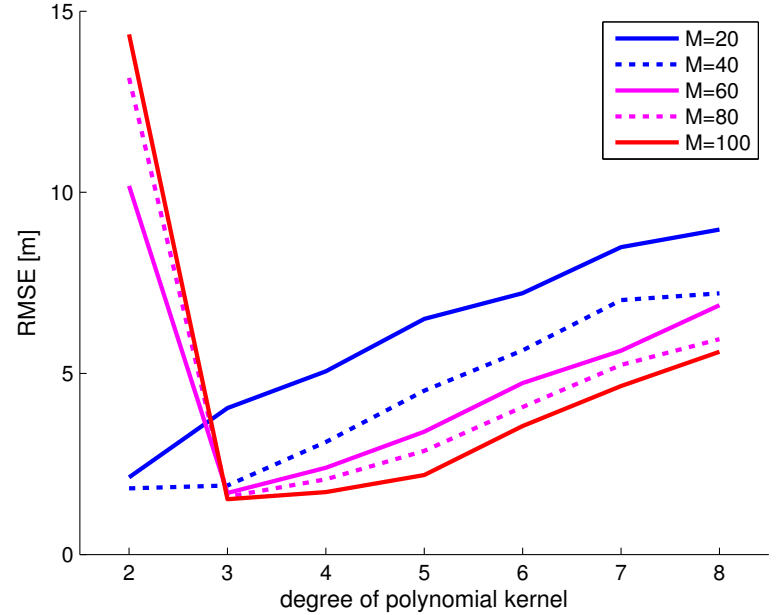

Fig. 3: RMSE as a function of degree of polynomial kernel (c) for different number of retained principal components $(M)$.

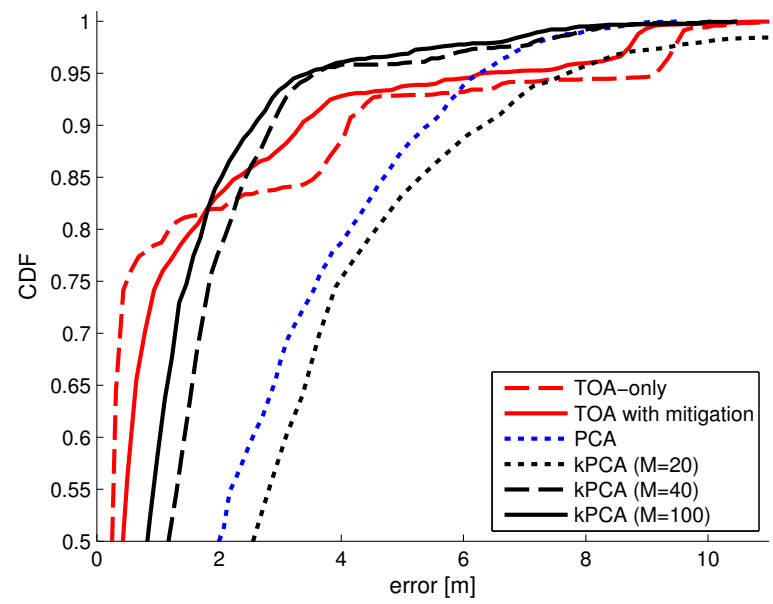

Fig. 4: $\mathrm{CDF}$ of the ranging error for different ranging algorithms.

worse for LOS samples. The gain is small because the exponential distribution used for NLOS identification does not perfectly match the real model [4].

- kPCA with $M=100$ is consistently the best approach for any percentile above 80th, since all principal components with non-negligible eigenvalues are used. kPCA with $M=40$ provides a similar performance. In both cases, the 95th percentile of the error is about $3 \mathrm{~m}$ lower comparing with TOA with mitigation. However, kPCA with smaller $M$ (e.g., $M=20$ ) would lead to a substantial loss in performance, and may perform even worse than linear PCA.

To summarize, kPCA with polynomial kernel and $40<M<$ 100 should be used for accurate ranging as long as low computational complexity is not required. Otherwise, TOA with mitigation is a good low-cost alternative.

\section{CONCLUSIONS}

We proposed a novel kernel-based technique for UWBbased ranging, and tested it using real data from a tunnel environment. Comparing with PCA and two TOA-based techniques, proposed kPCA technique (with polynomial kernel and enough retained principal components) provides much better ranging performance, especially in terms of maximum error. Therefore, it is useful for all positioning applications (such as search-and-rescue) in which there are enough computational resources. For the future work, we will try to improve the performance and efficiency of kPCA by using different kernel functions. Moreover, we plan to compare kPCA with other kernel-based machine learning techniques, such as Gaussian process regression.

\section{ACKNOWLEDGMENT}

This work was performed in the COOPLOC project supported by the Swedish Foundation for Strategic Research (SSF) and ELLIIT. We also thank Per Ängskog, and José Chilo (University of Gävle) for their help during the measurement campaign.

\section{REFERENCES}

[1] S. Gezici, Z. Tian, G. B. Giannakis, H. Kobayashi, A. F. Molisch, H. V. Poor, and Z. Sahinoglu, "Localization via ultra-wideband radios: a look at positioning aspects for future sensor networks," IEEE Signal Processing Magazine, vol. 22, pp. 70-84, July 2005.

[2] J. Khodjaev, Y. Park, and A. S. Malik, "Survey of NLOS identification and error mitigation problems in UWB-based positioning algorithms for dense environments," Annals of telecommunications, vol. 65, no. 5-6, pp. 301-311, 2010.

[3] B. Scholkopf, A. J. Smola, and K.-R. Muller, Advances in kernel methods, ch. Kernel principal component analysis, pp. 327-352. Cambridge, MA, USA: MIT Press, 1999.

[4] V. Savic, J. Ferrer-Coll, P. Angskog, J. Chilo, P. Stenumgaard, and E. G. Larsson, "Measurement analysis and channel modeling for TOA-based ranging in tunnels." available on http://arxiv.org/pdf/1312.6415v1.pdf, 2013.

[5] M. P. Wylie and J. Holtzman, "The non-line of sight problem in mobile location estimation," in Proc. of 5th IEEE Int. Universal Personal Communications Record. Conf, vol. 2, pp. 827-831, 1996.

[6] S. Venkatesh and R. Buehrer, "Non-line-of-sight identification in ultrawideband systems based on received signal statistics," IET Microwaves, Antennas \& Propagation, vol. 1, no. 6, pp. 1120-1130, 2007.

[7] J. Zhang and E.-S. Lohan, "Analysis of kurtosis-based LOS/NLOS identification using indoor MIMO channel measurement," IEEE Transactions on Vehicular Technology, vol. 62, pp. 2871-2874, July 2013.

[8] S. Marano, W. Gifford, H. Wymeersch, and M. Win, "NLOS identification and mitigation for localization based on UWB experimental data," IEEE Journal on Selected Areas in Communications, vol. 28, pp. 1026 1035, Sept. 2010.

[9] S. Gezici, H. Kobayashi, and H. V. Poor, "Non-parametric non-lineof-sight identification," in Proc. of IEEE Vehicular Technology Conf., vol. 4, (Orlando, FL), pp. 2544-2548, Oct. 2003.

[10] S. Al-Jazzar, J. Caffery, and H.-R. You, "A scattering model based approach to NLOS mitigation in TOA location systems," in Proc. of IEEE Vehicular Technology Conf. (VTC), vol. 2, pp. 861-865, 2002.

[11] B. Denis, J. Keignart, and N. Daniele, "Impact of NLOS propagation upon ranging precision in UWB systems," in Proc. of IEEE Conf. on Ultra Wideband Systems and Technologies, pp. 379-383, 2003.

[12] L. Cong and W. Zhuang, "Non-line-of-sight error mitigation in mobile location," in Proc. of IEEE INFOCOM, p. 650 659, 2004.

[13] H. Wymeersch, S. Marano, W. M. Gifford, and M. Z. Win, "A machine learning approach to ranging error mitigation for UWB localization," IEEE Trans. on Communications, vol. 60, pp. 1719 -1728, June 2012.

[14] D. Dardari, A. Conti, U. Ferner, A. Giorgetti, and M. Z. Win, "Ranging with ultrawide bandwidth signals in multipath environments," Proceedings of the IEEE, vol. 97, no. 2, pp. 404-426, 2009.

[15] C. M. Bishop, Pattern Recognition and Machine Learning. Secaucus, NJ, USA: Springer-Verlag New York, Inc., 2006. 